\title{
REAL-TIME CONTACT FORCE MEASUREMENT SYSTEM FOR PORTABLE COORDINATE MEASURING ARMS
}

\author{
GONZALEZ - MADRUGA, D[aniel]; CUESTA, E[duardo]; BARREIRO, J[oaquin]; \\ MARTINEZ PELLITERO, S[usana] \& ALVAREZ, B[raulio] J.
}

\begin{abstract}
This paper presents a real-time force measuring system development and implementation for Articulated Arm Coordinate Measuring Machines (AACMMs). Due to the lack of studies on the AACMM field, force analysis of touch trigger probes on Coordinate Measuring Machines (CMMs) are used to propose a new force measuring instrument. AACMM forces are measured with strain ganges on the hard probe of the AACMM. Strain gauges readings are used to calculate forces that operator causes during part measuring. The whole system has been calibrated according to international standards. A measuring contact force characterization is also carried out in order to know the measurement contact force influence in AACMM performance.
\end{abstract}

Keywords: coordinate measurement arms, AACMM, measurement contact force, traceability

\section{INTRODUCTION}

During last decade, AACMMs have experienced a great growth in the industry, mainly due to their great flexibility, portability, easy handling and reduced cost compared to CMMs. In contrast, AACMMs present a lower accuracy level but they are suitable for inspection tasks where CMMs precision level is not required. In addition, part transportation drawbacks arise in inspection and assembly operations when dealing with large and/or complex parts. These difficulties can be easily overcome with AACMMs.

Nevertheless, in spite of the AACMMs acceptance and the wide range of tasks they can fulfil, very few studies have been developed in order to increase the knowledge about their metrological behaviour and reliability. The studies are mostly focused on AACMMs calibration. AACMMs are physically defined by a group of parameters according to a kinematic model which determines the probe coordinates referred to a global fixed reference system. The calibration process calculates these parameters in order to minimize error produced when measuring a master piece. Santolaria et al. [1] optimize the kinematical model parameters in terms of volumetric accuracy and point repeatability by means of a non-linear least squares method. Calibration is also performed with annealing algorithms [2] to provide a better repeability. The proposed calibration process optimizes the AACMM global error but characterization, minimization o elimination of single error is not aimed.

Even fewer papers are focused in analysing AACMMs error sources. In a later work, Santolaria et al. [3] add a temperature error model to its calibration method which adjusts the kinematic model parameters and provides more reliability in field measurements, where temperature is not controlled and therefore enhancing their flexibility. Deflection of AACMMs structure is studied by Vhrovec [4] by locating optic sensors inside the largest segments of the AACMM. This method corrects the parameters corresponding to segments length. However, this method is incompatible with the optimization of the calibration method because it changes parameters without taking into account the physical meaning of the kinematical model parameters. Furthermore, this method has not been implemented on probes.

Despite only previous error sources have been corrected, Vhrovec stated that deflection provoked by force during part measurement consitutes one of the main error source [4]. It is also noted in previous work [5] that AACMM error is heavily influenced by the measuring force applied by operators.

Moreover, only two standards deal with AACMMs [6][7] and focused in calibration. Rigorous calibration processes are defined but no guidance is offered to the operator apart from trying to reach specific AACMM postures. Furthermore, calibration is usually performed with special probes such us the proposed by Santolaria [1] which leads to greater errors when other probes are used.

Because of these reasons, this paper is aimed to characterize measuring force on the probe. Force and probe knowledge constitute two differences between CMMs and AACMMs. With regard to CMMs, force is completely controlled by configurable parameters in the CMM software. In addition, touch trigger probes increase this control since the force applied is well-known. Touch trigger probes read the contact point when a circuit opens by the effect of the measuring force. Both the force and the probe displacement, pretravel, are limited and they are used to correct the measured point coordinates. However this kind of probes are not common in contact measuring with AACMMs.

Measuring force in touch trigger probes is studied from different points of view depending on author's criteria. In Pereira work [8] transducers are used in order to characterize touch trigger probes error. Transducers are in contact with the part and aligned with CMM axes, so measuring forces are defined in the CMM reference coordinate system instead of the probe coordinate system. This method is very useful for CMMs where every parameter, such as probe direction or contact point, is known with a high accuracy and repeability level and, therefore, force in probe coordinate system could be calculated and used to work out the probing error. In the AACMMs case, relative position between part and probe 
requires a more complex process. In addition, the time of point reading is manually controlled by operator.

Other authors [9][10] characterize touch trigger probes by measuring their error without being installed in the CMM. Once probe error is obtained it is used to compensate and analyze its behaviour. However, since probes work together with CMMs their performance affects each other. This facts is even more important with AACMMs due to the lack of knowledge about their behaviour. Therefore, studies on ACCMMs and probes shall be carried out taking into account their relationship.

Another option consists in using strain gauges directly mounted on the AACMM probe, as Liang [11] states in its probe design. Strain gauges allow force measuring in the probe coordinate system. Furthermore, they do not change probe geometry so real behaviour is measured. Triaxial sensors are also available but they involve probe geometry changing and their cost is very high.

Due to the aforementioned reasons, in this work a new method is proposed to characterize force during AACMMs measuring by means of strain gauges. It is also studied the signal processing in order to be able to analyse the force. A new force measuring instrument adapted to metrological characteristics of AACMMs (in contact with the probe) is designed and implemented. The final objective of the instrument is to collect the data required to characterize force on real-time and, subsequently, to minimize or compensate its effects.

As part of this work calibration of the whole system is performed according to the GUM standard [12]. Once calibration is done, measuring force is recorded at any time and study of one of the main error sources of AACMMs is performed. An experimental test is proposed in order to characterize the force behaviour during the measurement of a real part.

Future works include a in-deep force analysis, the elaboration of force compensation models and the generation of an operator manual in order to reduce the force influence and increase the AACMM reliability.

\section{FORCE MEASURING DESIGN}

After analyzing several options for measuring force, strain gauges were selected as the more suitable option in order to keep the true geometry of the probe. A Romer Sigma 2018 with $1.8 \mathrm{~m}$ measuring range and 6 degrees of freedom with a hard probe for contact measuring were used.

The proposed system consists in three phases: design and implementation of strain gauges, force signal acquisition and data processing. The AACMM measuring process with this system adds one more phase: AACMM measurement, force measurement (both at the same time), data acquisition and data processing (Fig.1).

AACMM measurement phase is performed as a usual measurement. At the same time that operator probes a point, the system measures the force applied by the operator. The signal created by the force and collected by the strain gauges is acquired and primarily processed with the acquisition instrument software. These data are sent to proper data processing software, Matlab $^{\circledR}$ in this case, and converted into the measuring force. Finally both metrologic and force information is obtained thanks to the AACMM and the proposed system.

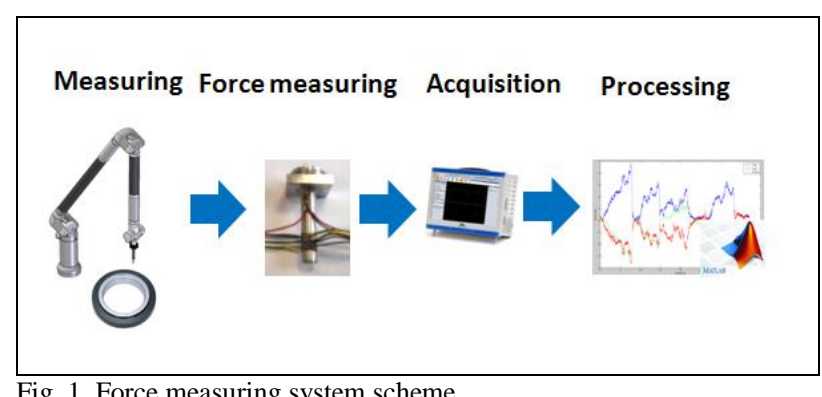

In the following sections the system design phases are presented.

\section{Design and implementation}

A wide range of probes is used on CMMs, touch trigger probes mostly, and they have been fully researched. However, AACMMs commonly use hard probes. Hard probes are basically a rigid cylinder with a sphere at one extreme. These probes are not equipped with any error compensation or contact detection system. Fig. 2 shows the hard probes supplied by default by the manufacturer: a $15 \mathrm{~mm}$ steel sphere probe used in probe calibration before measuring, a $6 \mathrm{~mm}$ ruby sphere $45 \mathrm{~mm}$ long probe and finally, a point (sharp-needle ended) 85 $\mathrm{mm}$ long probe. A $6 \mathrm{~mm}$ ruby sphere $85 \mathrm{~mm}$ long probe was acquired additionally.

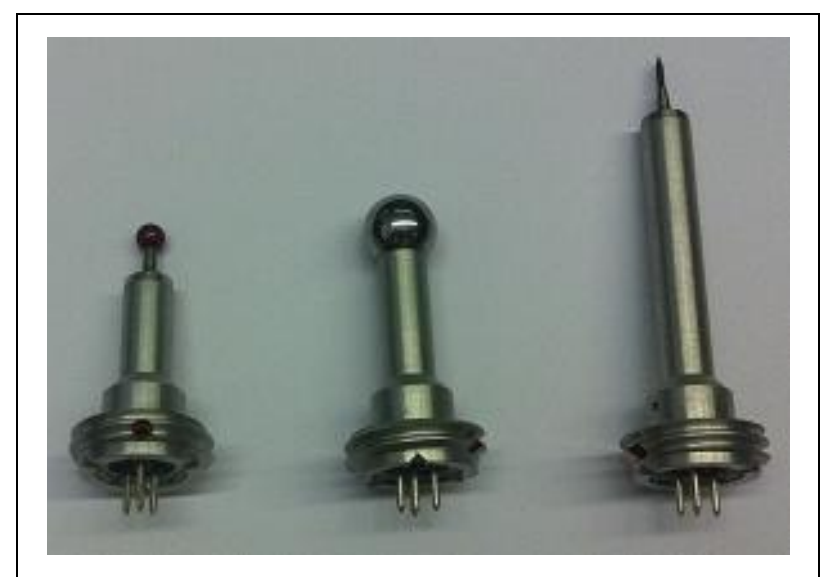

Fig. 2. Standard AACMMs probes (ROMER Sigma \& Omega brands)

The $6 \mathrm{~mm}$ sphere $85 \mathrm{~mm}$ long probe was chosen to implement the strain gauges on. It is composed of an aluminium cylinder body $85 \mathrm{~mm}$ long and $10 \mathrm{~mm}$ of diameter and a $6 \mathrm{~mm}$ diameter ruby sphere at the end. Other probe configurations are also suitable for the instrument design if strain gauge size allow it.

Measuring force appears as a result of the contact between probe and part by the operator action. This force is normal to the part surface and it is applied in the sphere at the contact point. It varies from one point to another since it depends on the operator criteria while 
ensuring the contact. In addition, it is difficult to manually keep a constant force level especially when uncomfortable measuring postures are necessary to be able to reach a point. Measuring force is composed of components referred to the probe coordinate system $F_{x}$, $F_{y}$ and $F_{z}($ Fig 3$) . F_{x}$ and $F_{y}$ are perpendicular to the probe axis and $F_{z}$ is parallel.

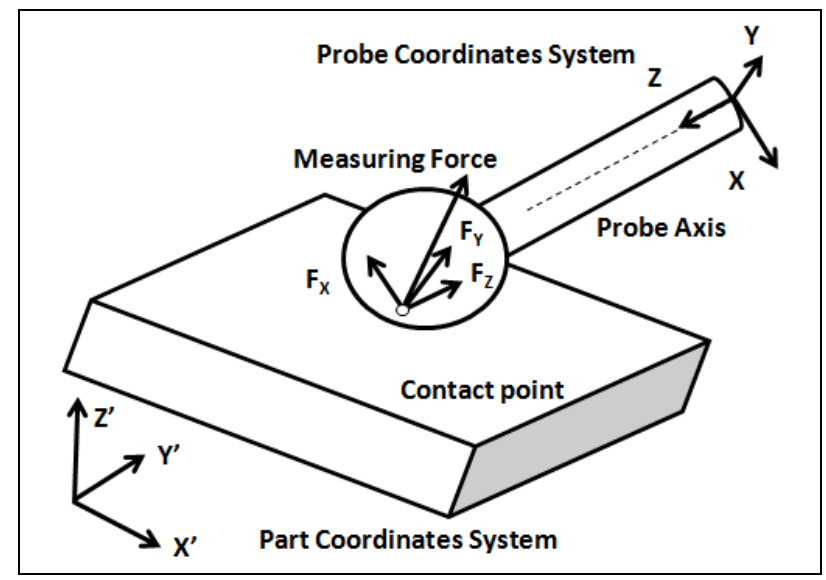

Fig. 3. AACMM measuring forces

As a result, $\mathrm{F}_{\mathrm{z}}$ is a compression force that causes a reduction of the probe length and $F_{x}$ and $F_{y}$ are bending forces that cause a probe deflection.

When these three components $\left(F_{x}, F_{y}, F_{z}\right)$ are measured, the total force and its direction can be calculated. When one component is not measured, a partial total force and its direction within the plane generated by the measured forces can be figured out. This option is suitable for probes with limited surface as in this case. Furthermore, two forces provide enough information for their characterization. Additionally, comparison between operators can be made since they are under the same conditions.

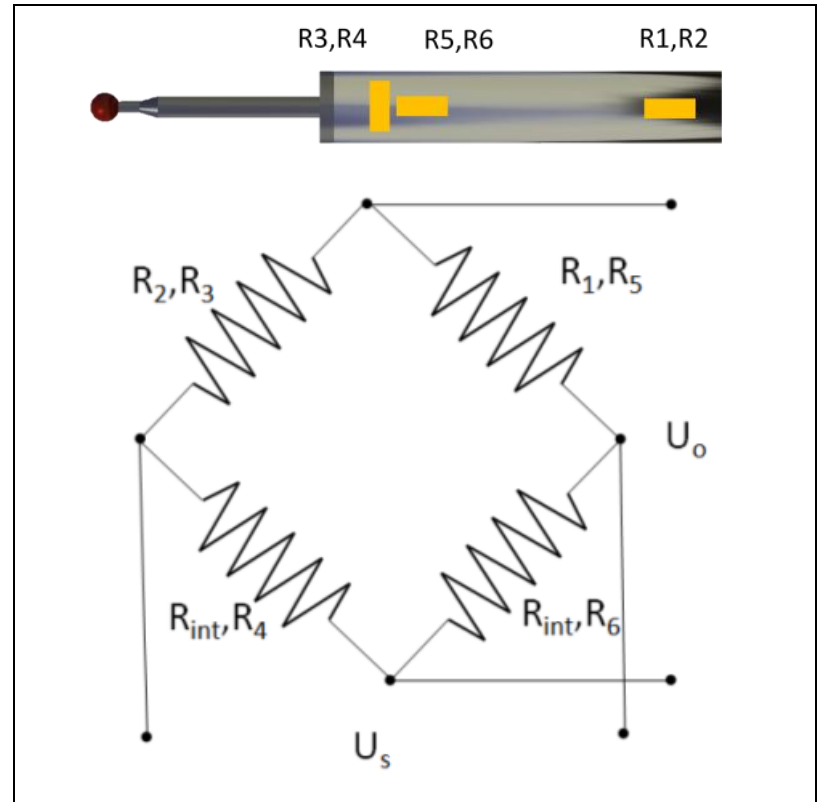

Fig. 4. Configuration of the bridge circuit.

Fig. 4 shows the strain gauges arrangement on the probe and the bridge circuit for force reading. The arrangement is repeated on the other side of the probe. $F_{z}$ and $F_{x} / F_{y}$ signals are read from different circuits. Strain gauges R3, R4, R5 and R6 are used to detect $F_{z}$ with a full bridge circuit. Strain gauges R1 and R2 are used to detect $F_{x} / F_{y}$ with a half bridge circuit completed with Rint in the acquisition instrument. The probe deformation causes a change in the value of the output voltage of the bridge circuit. This output is proportional to the strain generated.

Technical specifications of strain gauges used in the circuit are shown in Tab.1.

\begin{tabular}{|c|c|c|}
\hline Strain gauge & Nominal resistance [ $\boldsymbol{\Omega}]$ & Resistance name \\
\hline 1-LY13-3/350 & 350 & R1,R2 \\
\hline 1-XY13-3/350 & 350 & R3,R4,R5,R6 \\
\hline
\end{tabular}

Once designed the gauges arrangement, the force measuring system is implemented on the probe as shown in Fig. 5. The output signal is collected in the next phase.

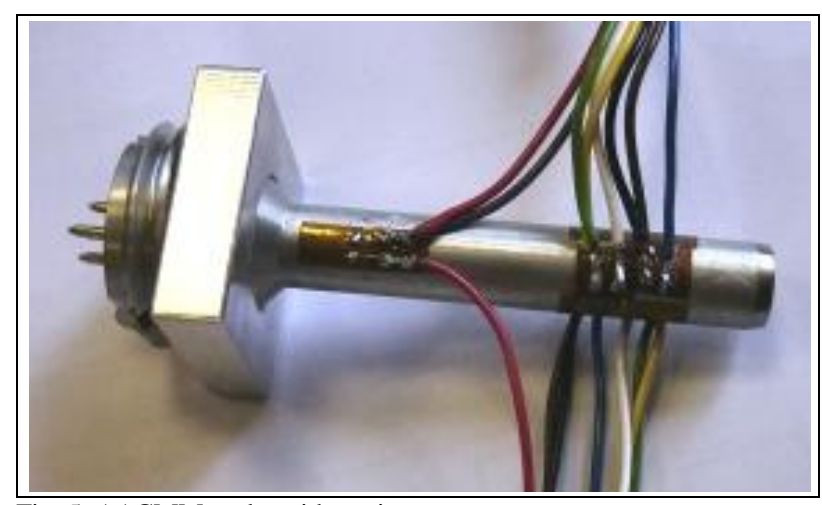

Fig. 5. AACMM probe with strain gauges

\section{Signal acquisition}

The signal sent from the bridges is collected by a data acquisition system, specifically a Dewetron 3021 (Fig. 6). This instrument allows to configure the signal and to perform a primary processing. The signal is captured at $1000 \mathrm{~Hz}$. In addition, the Dewesoft $\mathrm{C}$ control software is capable of adjusting the signal to specific levels in order to match the 0 signal with 0 load and the maximum signal with the maximum load.

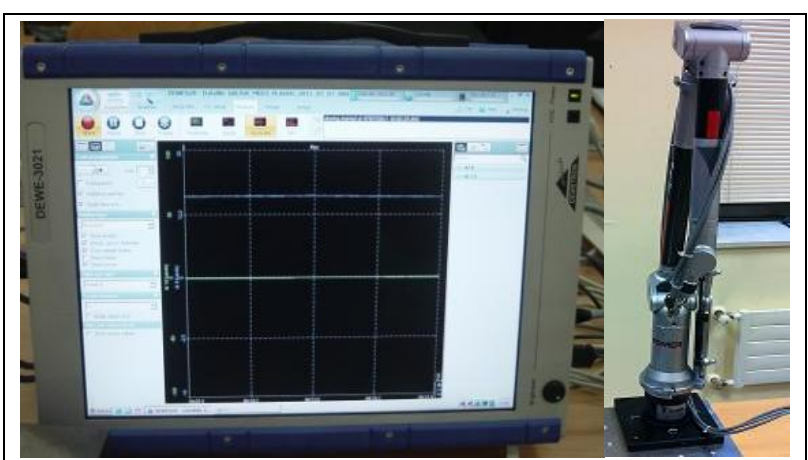

Fig. 6. Data acquisition instrument

The adjustment process connects a known applied weight with the signal received. The weights used were $0 \mathrm{~g}$ and $1000 \mathrm{~g}$. Common force applied while measuring is under $6 \mathrm{~N}$ but sometimes it goes above $10 \mathrm{~N}$, particularly 
when using AACMM higher that $2.5 \mathrm{~m}$ long or when operator posture is uncomfortable.

\section{Signal processing}

Collected signal is saved in Matlab ${ }^{\circledR}$ format in order to make the subsequent process easier. Knowing the load applied during the adjustment operation, the signal is converted into the measuring force. Fig.7 presents the results from this phase.

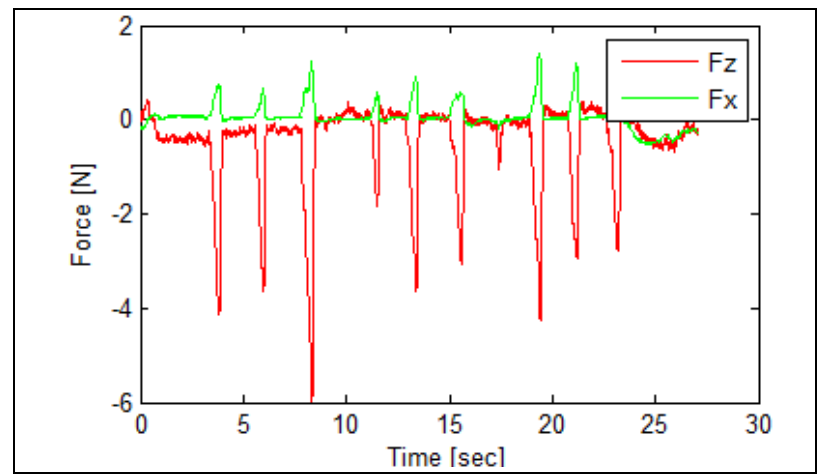

Fig. 7. Graphic of measuring force

From the analysis of this kind of graphics a valuable knowledge can be extracted in order to characterize the AACMM performance.

Once the system is implemented, the associated uncertainty must be established by means of a calibration process, as explained in the next section.

\section{CALIBRATION}

GUM standard [12] was used to obtain the uncertainty of the force measuring system. The uncertainty of the whole system is composed of the uncertainty of all the error sources that affect the system. Calibration is carried out twice, for $F_{z}$ and $F_{x}$. Calibration tests have been performed with two weight ranges. The first one goes from $0 \mathrm{~g}$ to $200 \mathrm{~g}$ and the second one goes from $200 \mathrm{~g}$ to $1300 \mathrm{~g}$.

The whole system uncertainty is called combined uncertainty $\left(U_{\text {com }}\right)$. Combined uncertainty includes the following components: standard weights uncertainty $\left(U_{s t w}\right)$, creep uncertainty $\left(U_{c r e}\right)$, zero uncertainty $\left(U_{E o}\right)$, resolution uncertainty $\left(U_{\text {res }}\right)$, hysteresis uncertainty $\left(U_{\text {hys }}\right)$, repetibility uncertainty $\left(U_{\text {rep }}\right)$ and temperature uncertainty $\left(U_{\text {tem }}\right)$. It is calculated as follows (1):

$$
\begin{aligned}
& U_{\text {com }}=\sqrt{\sum U_{i}^{2}}= \\
& \sqrt{{U_{s t w}}^{2}+U_{c r e}{ }^{2}+{U_{E_{0}}}^{2}+{U_{r e s}}^{2}+{U_{h y s}}^{2}+U_{r e p}{ }^{2}+U_{t e m}{ }^{2}}
\end{aligned}
$$

Each uncertainty component is obtained from calibration certificates or statistical results obtained from tests. Combined uncertainty is multiplied by a constant (k) which value depends on the level of confidence to obtain the expanded uncertainty $\left(U_{\text {exp }}\right)(2)$ :

$$
U_{\text {exp }}=k U_{\text {com }}
$$

where $\mathrm{k}$ is equal to 2 for a $95 \%$ coverage level.

Test results for each uncertainty component are shown in Tab.2. Uncertainties have associated

\begin{tabular}{|c|c|c|}
\hline \multirow{2}{*}{$\begin{array}{l}\text { Uncertainty } \\
\text { component }\end{array}$} & \multicolumn{2}{|c|}{ Uncertainty $(0-200 \mathrm{~g} / 200-1300 \mathrm{~g}$ range) } \\
\hline & $\mathrm{Fz}[\mathrm{N}]$ & $F x[N]$ \\
\hline$U_{s t w}$ & $0,0012 / 0.0042$ & $0,0012 / 0.0042$ \\
\hline$U_{\text {cre }}$ & $0.002 / 0.008$ & $0.002 / 0.008$ \\
\hline$U_{E o}$ & 0.003 & 0.008 \\
\hline$U_{\text {res }}$ & 0.001 & 0.001 \\
\hline$U_{\text {hys }}$ & 0.055 & 0.038 \\
\hline$U_{\text {rep }}$ & 0.046 & 0.025 \\
\hline$U_{\text {tem }}$ & 0.000 & 0.000 \\
\hline$U_{\text {com }}$ & $0.072 / 0.072$ & $0.046 / 0.047$ \\
\hline$U_{\text {exp }}$ & $0.144 / 0.144$ & $0.092 / 0.094$ \\
\hline
\end{tabular}

probabilistic distributions that allow to calculate the combined uncertainty at the coverage level mentioned.

Tab. 2. Calibration uncertainty results

Expanded uncertainty was $0.144 \mathrm{~N}$ for $\mathrm{F}_{\mathrm{z}}$ and 0.092 $\mathrm{N} / 0.094 \mathrm{~N}$ for $\mathrm{F}_{\mathrm{x}}$ for the weight ranges 0-200/200$1300 \mathrm{~g}$, respectively.

\section{FORCE CHARACTERIZATION}

At this stage, force can be registered and analyzed in order to study its influence on the AACMM performance and to characterize the force and operator behaviour. The following experimental tests have been carried out. An artefact (reference part) has been measured with the AACMM and with the force measuring system simultaneously. The artefact includes several planes with different orientations, Fig. 8. A total of three operators have measured the planes and each operator has repeated the test three times.

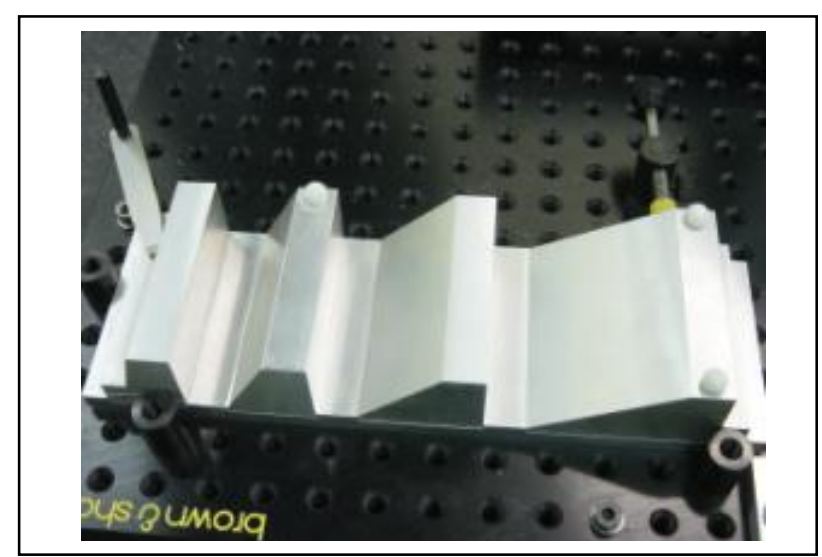

Fig. 8. Artefact measured in the experimental tests

In order to keep the rest of parameters constant, with the exception of the force and operator, contact point distribution (number of points per element and its distribution) was previously determined. For achieving a similar distribution of contact points, guiding marks were used to show to the operator the number of points and their location approximately.

Apart from this, no measuring guidance was given to operators. Each operator followed their own criteria and strategy for measuring (measuring time, probe orientation, level of force, their posture, AACMM posture, "point to point" measuring or "continuous touching" measuring, etc.).

Therefore, this methodology let characterize different types of measuring, which can be analyzed and compared. Manual control of AACMM causes a wide 
range of measuring results. By identifying and studying them AACMM measuring strategies can be developed. CMMs, automatically controlled, have a considerable knowledge in this field but it is not directly applicable to AACMMs because of their different kinematic behaviour, the manual control and the lack of studies of AACMMs.

Once experimental tests have been performed and force results have been obtained, a comparison among repetitions and operators results was made. Fig. 9. shows the most representative measuring force graphics obtained. In this graphic the first row corresponds to repetitions for the same operator whereas the second row corresponds to two different operators with different strategies. As can be noted, $F_{z}$ and $F_{x}$ are shown along the experimental test duration in red and green lines, respectively.

Knowledge can be extracted from force results. Firstly, it is obviously noted that measuring time changes between different repetitions and between different operators. As for the force signal itself, its shape also change but contact points are easily identified since they coincide with the instant of maximum or minimum force, depending on the probe orientation. There are as many peaks (maximum/minimum) as points measured.

To characterize force behaviour some parameters are defined:

1. Force level: Operators tend to ensure contact by pressing the part with the probe, but contact perception varies from operators. Force level, indicated by maximum force, changes completely
2. between operators $\left(\mathrm{F}_{\mathrm{z}}\right.$ is around $1.5 \mathrm{~N}$ in a.2 case and above $10 \mathrm{~N}$ in b. 1 case) and also between repetitions of the same operator (around $1.5 \mathrm{~N}$ in a.2 case and 6 $\mathrm{N}$ in a.1 case). A higher force level could lead to greater errors.

3. Force variability: manual control causes a variation on the force during measuring. Force level, indicated by minimum and maximum forces, changes between operators (1-1.5 $\mathrm{N}$ in a. 2 case and $5-10 \mathrm{~N}$ in b. 1 case) and between repetitions of the same operator (1-1.5 N in a. 2 case and 1-6 $\mathrm{N}$ in a.1 case). A non-uniform force distribution could lead to a non-uniform error distribution.

Apart from force parameters two types of force curves are identified according to the measuring strategies: point to point measuring (a.1, a.2 and b.1 cases) and continuous measuring (b.2 case).

1. In point to point measuring strategy operators eliminate the contact between part and probe after point reading. Therefore, measuring force returns to $0 \mathrm{~N}$ (a.1, a.2 and b.1 cases).

2. In continuous measuring contact is kept. Therefore there is not back to $0 \mathrm{~N}$ force (b.2 case).

Another interesting point is the relation found between $F_{x}$ and $F_{z}$. This parameter indicates if the measuring is carried out perpendicular or parallel to the surface, or at any other intermediate orientation. If $\left.\mathrm{F}_{\mathrm{z}}\right\rangle$ $\mathrm{F}_{\mathrm{y}}$ measuring tends to be perpendicular whereas if $\mathrm{F}_{\mathrm{z}}<\mathrm{F}_{\mathrm{y}}$ measuring tends to be parallel.

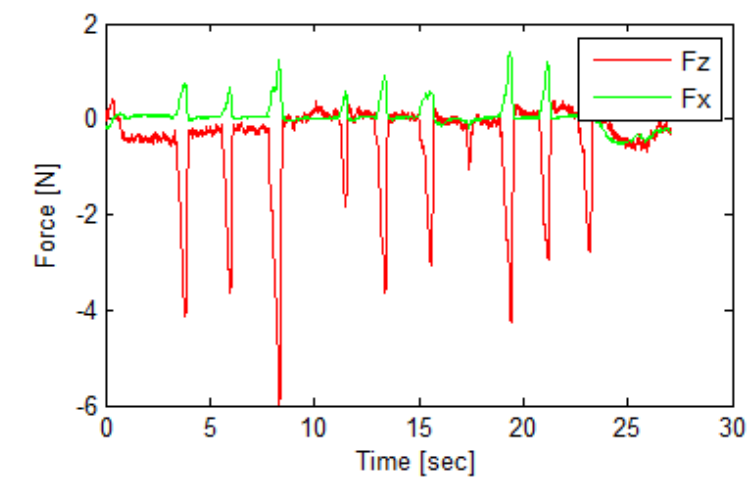

a.1) Operator 1, Measurement 1

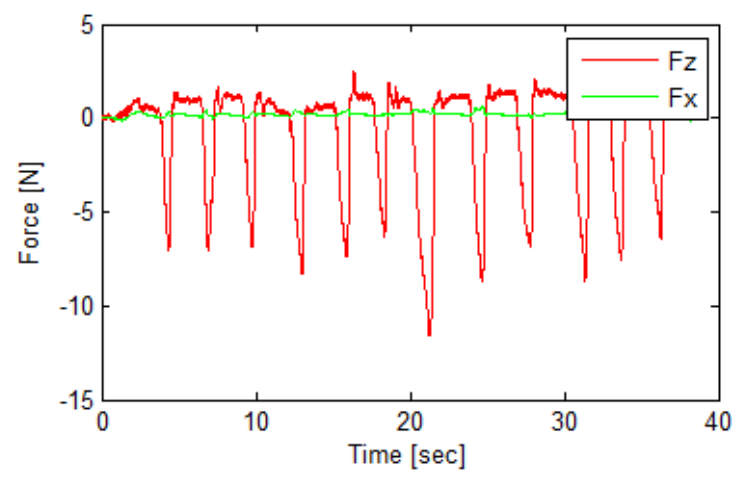

b.1) Operator 2, Measurement 1

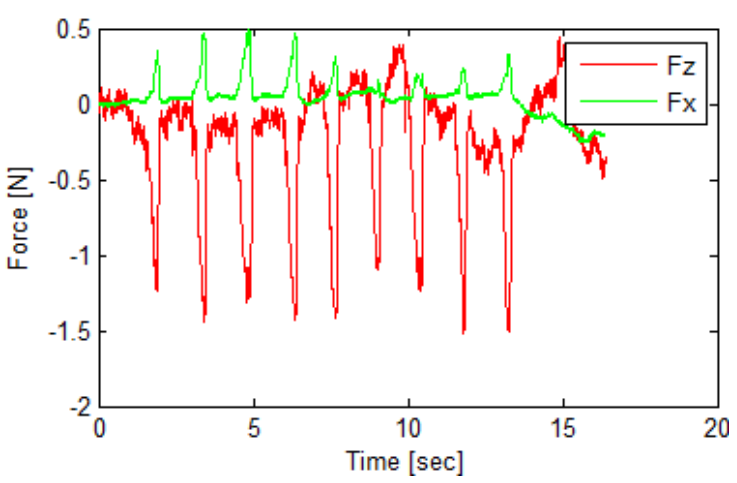

a.2) Operator 1, Measurement 2

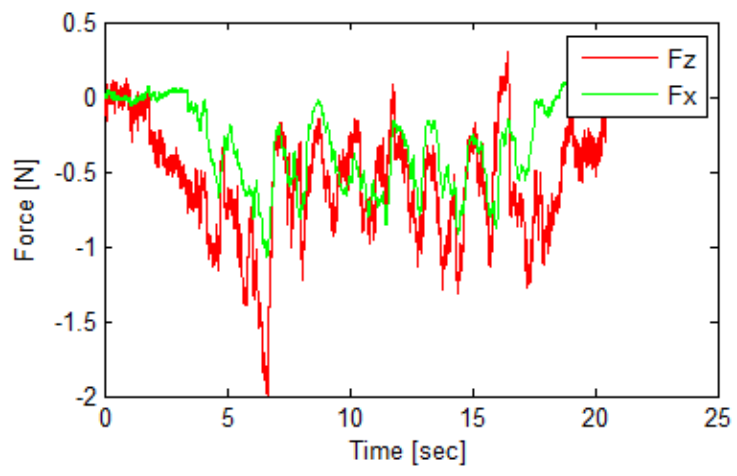

b.2) Operator 3, Measurement 1 


\section{CONCLUSIONS}

A real-time force measuring system has been designed and implemented for detecting measuring contact forces acting upon AACMMs. The system has been calibrated according to GUM standard [12]. The system allows to analyze the influence of measuring force over the precision of AACMMs.

Experimental tests have been carried out and measuring forces have been characterized. A force analysis shows that force level and its variability changes significantly among measurement repetitions and operators. Also, different measuring strategies for measuring planning have been identified. It has been appreciated that operator, applied force and measuring strategies have an important influence on AACMMs performance. Therefore, further studies are required on the matter to give solution to these error sources.

Future work includes a in-deep study of measuring forces and the preparation of guidance strategies for a proper measurement with AACMMs. It is also possible to elaborate compensation models aimed to improve the AACMM accuracy and reliability. Furthermore, conclusions can be used to develop KBE systems in order to define a proper measuring process.

\section{ACKNOWLEDGEMENTS}

Authors gratefully acknowledge the support provided by the Instituto Universitario de Tecnología Industrial de Asturias (IUTA) through the project entitled: "Estudio y desarrollo de modelos de optimización para la cualificación de brazos portátiles de medir por coordenadas" (ref. SV-12-GIJON-1). Also, to the Spanish Ministry of Economy and Competitiveness for the financial support through the project "Aseguramiento de la calidad y representacion del conocimiento en la medicion con sistemas portatiles de medir por coordenadas" (ref. DPI2012-36642-C02-01). Finally, to the Sociedad de Ingeniería de Fabricación (SIF) for the mobility grant that allowed to finish this research.

\section{REFERENCES}

[1] Santolaria J, Aguilar JJ, Yagüe JA, Pastor J. (2008). Kinematic parameter estimation technique for calibration and repeatability improvement of articulated arm coordinate measuring machines. Available from: www.sciencedirect.com/science/article/pii/ S0141635907001146 Accessed on: 2012-07-20

[2] Guanbin Gao, Wen Wang, Keng Lin and Zichen Chen. (2009). Structural Parameter Identification for Articulated Arm Coordinate Measuring Machines. International Conference on Measuring Technology and Mechatronics Automation. Available from:http://ieeexplore.ieee.org/xpl/articleDetails.jsp?reload= true \&arnumber $=5203393 \&$ content $T y p e=$ Conference + Publication s Accessed on: 2012-07-20

[3] Jorge Santolaria and José-Antonio Yagüe and Roberto Jiménez and Juan-José Aguilar (2009). Calibration based thermal error model for articulated arm coordinate measuring machines. Available from: http://www.sciencedirect.com/science/article/ pii/S0141635909000312 Accessed on: 2012-07-20
[4] Vrhovec, M. and Munih, M. (2007). Improvement of coordinate measuring arm accuracy. Available from: http://ieeexplore.ieee.org/xpl/login.jsp?tp=\&arnumber $=4399098 \&$ url=http $\% 3$ A\%2F\%2Fieeexplore.ieee.org\%2Fiel5\%2F4398943\% 2F4398944\%2F04399098.pdf\%3Farnumber\%3D4399098 Accessed on: 2012-07-20

[5] Cuesta, E. Álvarez, B.J. Martínez, S. Barreiro, J. GonzálezMadruga, D. (2011) Evaluation of influence parameters on measurement reliability of Coordinated Measuring Arms. Available from: http://proceedings.aip.org/resource/2/apcpcs/ 1431/1/217_1 ?isAuthorized=no Accessed on: $2012-07-20$

[6] ASME B89.4.22-2004. (2004) Methods for performance evaluation of articulated arm coordinate measuring machines, American Society of Mechanical Engineers. Available from: http://www.asme.org/products/codes---standards/methods-forperformance-evaluation-of-articulated -Accessed on: 2012-07-20

[7] VDI/VDE 2617 Part 9. (2009) Acceptance and reverification test for articulated arm coordinate measuring machines, Verein Deutscher Ingenieure. Available from: http://www.vdi.eu / Accessed on: 2012-07-20

[8] [Per07] Pereira, P.H.; Hocken, R.J. Characterization and compensation of the dynamic errors of a scanning coordinate measuring machine. (2007). Available from: http://www.sciencedirect.com/science/article/pii/S014163590600 047X Accessed on: 2012-07-20

[9] Woźniak,A.; Dobosz,M.(2003) Metrological feasibilities of CMM touch trigger probes. Part I 3D theoretical model of probe pretravel. Available from: http://www.sciencedirect.com/science/ article/pii/S026322410300071X Accessed on: 2012-07-20

[10] Dobosz,M.; Woźniak,A. (2003) Metrological feasibilities of CMM touch trigger probes Part II Experimental verification of the 3D theoretical model of probe pretravel. Available from: http://www.sciencedirect.com/science/article/pii/S026322410300 0721 Accessed on: 2012-07-20

[11] Liang,Qiaokang; Zhang,Dan; Wang,Yaonan; Ge,Yunjian. (2012). Development of a touch probe based on five-dimensional forcetorque transducer for coordinate measuring machine (CMM). Available from: http://dl.acm.org/citation.cfm?id=2051526 Accessed on: 2012-07-20

[12] ISO GUM. Guide to the expression of uncertainty in measurements. Geneva: International Organization for Standardization - ISO; 2008. Available from: http://www.bipm.org/en/publications/guides/gum.html Accessed on: 2012-07-20 